\title{
LASER PROBING OF THE PLASMA IN THE S-300 FACILITY
}

\author{
Yu.G. Kalinin, V.A. Korel'skii, E.V. Kravchenko, and A.Yu. Shashkov \\ Russian Research Center "Kurchatov Institut", Kurchatov Square 1, \\ Moscow 123182, Russia
}

PACS: 52.70.-m

1. The S-300 eight-module generator, operating at a current of up to $4 \mathrm{MA}$ with a current rise time of $100 \mathrm{~ns}$ and impedance of $0.15 \mathrm{Ohm}$, is destined for experiments with high-temperature pulsed plasmas of light liners and Z-pinches. Among a number of different diagnostics the facility has been equipped by laser diagnostic setup too.

Laser probing (interferometry, shadow and the schlieren photography, etc.) has long been a classic method for diagnosing imploding plasmas. This method allows one to study a plasma with a definite density magnitude and gradients. However, to implement this diagnostics in pulsed high-current generators, it is necessary to solve a number of problems related to both the design of the generators and the specific conditions under which experiments are carried out: large distances between the probing radiation source, the object under study, and the image plane; the relatively small dimensions of the object; and, finally, rigid requirements to the synchronization between the probing laser pulse and the current pulse through the load. In this paper, we describe a system for the laser probing of the plasma in the S-300 high-current generator [1]. In our opinion, the system is fairly well suited for these conditions.

2. The diagnostic setup included the transmitting and receiving laser components.

The receiving component, which was used to record the shadow images of the plasma was mounted in the facility room near the S-300 generator. It consisted of a lens $(\mathrm{f}=1600 \mathrm{~mm})$ imaging the plasma with a magnification factor of 2 and a camera equipped with a set of filters and a photographic cassette. Optical wedges with an angle of $1^{\circ}$ were positioned near the focal plane of the lens in order to separate the beams in the image plane by a required distance. If needed, different schlieren masks could be positioned in the focal plane of the lens. For immobile objects, the resolution of the system in the object plane was no worse than 35 line $/ \mathrm{mm}$.

The transmitting component was located in a separate room; the distance between the laser system and the target unit exceeded $18 \mathrm{~m}$.

The first version of the transmitting component (Fig.1) consisted of a YAG:Nd driving oscillator (DO), a threestage second-harmonic generator (SHO), and optical delay lines. An electrooptical shutter was synchronized with the current pulse of the S-300 generator. Each SHO stage consisted of a single-pass amplifier, a $\mathrm{LiNbO}_{3}$ nonlinear crystal with the temperature-sensitive tuning to the phase matching, and a Glan prism. After passing through the prism, second-harmonic radiation arrived at the optical delay line and, then, at a set of mirrors directing this radiation onto the plasma object. Radiation at the fundamental frequency, which was polarized orthogonally to the second-harmonic radiation, passed through the Glan prism without deflection. Then, it was amplified and used to generate the second harmonic in the next stage.

The energy in each probing beam was $20 \mathrm{~mJ}$, the wavelength was $532 \mathrm{~nm}$, the divergence was $\sim 1 \mathrm{mrad}$, the pulse duration was $10 \mathrm{~ns}$, and the interval between pulses was $25 \mathrm{~ns}$.

With this scheme for the probing-beam generation, all laser and nonlinear optical components operated under radiation loads that were far below the limiting ones. This substantially improved the reliability of the diagnostic setup operation, so that we could adjust the entire diagnostic system in the pulse-periodic mode with a repetition rate of about $1 \mathrm{~Hz}$. This is particularly important for experiments with large installations.

Figures 2 and 3 show plasma photographs of different S-300 loads.

To increase both time and spatial resolution, the second version laser setup has been elaborated (Fig.4). It included a Q-switch TEM 00 YAG:Nd DO with two laser amplifiers and a stimulated Brillouin scattering (SBS) compressor of the DO pulse. Phase conjugation and pulse compression in various nonlinear media in the course of SBS were studied in detail by many authors (see, e.g., [27]) and have already been used in plasma diagnostics [8]. The backward scattered beam was deflected by a polarizing deflector and was directed to a five secondharmonic generators similar to those mentioned above with $\mathrm{LiNbO}_{3}$ or KTP non-linear crystals. Secondharmonic pulse energy was about 3-5 mJ. Five secondharmonic beams were directed into S-300 output unit after passing their own delay lines. Thus, this assembly permitted to obtain five-frame shadow images of plasma loads with exposure of $1 \mathrm{~ns}$ and time delay between the frames of $10 \mathrm{~ns}$.

The diagnostic setup was adjusted in the pulse-periodic mode at a repetition rate of $0.25-0.5 \mathrm{~Hz}$.

Fig.5 shows shadow photographs of the different Zpinchs.

3. There are several reasons that may be responsible for the formation of a shadow in photographs.

The first reason is the cutoff of the probe radiation in plasma when the radiation frequency approaches the plasma frequency $\omega_{0}$ :

$$
\omega \approx \omega_{0}=\left(4 \pi \mathrm{N}_{\mathrm{e}} \mathrm{e}^{2} / \mathrm{m}\right)^{1 / 2}
$$

For a beam with a wavelength of $532 \mathrm{~nm}$, this occurs at densities of $\mathrm{N}_{\mathrm{e}} \sim 4 \cdot 10^{21} \mathrm{~cm}^{-3}$. 
Second, the probe radiation can be absorbed via inverse bremsstrahlung (i.e., due to free-free transitions). In this case, the attenuation of laser radiation is described by Bouguer's law,

$$
I_{v}=I_{v 0} \exp (-\theta \cdot 1)
$$

where 1 is the distance passed by the probing beam and the absorption coefficient $\theta$ is determined by the expression [9]

$$
\theta=\left(\left(\mathrm{C}_{1} \mathrm{Z}^{2} \mathrm{gN}_{\mathrm{e}} \mathrm{N}_{\mathrm{i}}\right) /\left(\mathrm{T}_{\mathrm{e}}{ }^{1 / 2} \mathrm{v}^{3}\right)\right) \cdot\left(1-\exp \left(-\mathrm{h} \mathrm{v} / \mathrm{kT}_{\mathrm{e}}\right)\right)
$$

For $\mathrm{kT}_{\mathrm{e}}>>\mathrm{hv}$, wich is always satisfied in our experiments, the absorption coefficient is described by the formula

$$
\theta \approx\left(\mathrm{C}_{1} \mathrm{~h} / \mathrm{k}\right) \cdot\left(\mathrm{ZgN}_{\mathrm{i}}^{2} / \mathrm{T}_{\mathrm{e}}^{3 / 2} v^{2}\right) .
$$

Here, $\mathrm{C}_{1}=3.69 \cdot 10^{8} \mathrm{~cm}^{-3} \cdot \mathrm{K}^{1 / 2} \mathrm{~s}^{-3}, \mathrm{Z}$ is the ion charge, $\mathrm{g}$ is the Gaunt factor, $\mathrm{N}_{\mathrm{e}}$ and $\mathrm{N}_{\mathrm{i}}$ are the electron and ion densities, respectively, $v$ is the probe-radiation frequency. From this formula, we obtain the following expression for $\mathrm{N}_{\mathrm{i}}$ :

$$
\mathrm{N}_{\mathrm{i}} \approx 7.5 \mathrm{v}\left(\left(\theta \mathrm{T}_{\mathrm{e}}^{3 / 2}\right) /\left(\mathrm{Z}^{3} \mathrm{~g}\right)\right)^{1 / 2}
$$

For the plasma under our conditions, we have $\mathrm{N}_{\mathrm{i}}>5 \cdot 10^{18} \mathrm{CM}^{-3}$.

It should be noted that, for a plasma of high $\mathrm{Z}$ materials, the ion density estimated by this formula depends weakly on the electron temperature. Indeed, we have $\mathrm{N}_{\mathrm{i}} \sim\left(\mathrm{T}_{\mathrm{e}}\right)^{3 / 4} /(\mathrm{Z}(\mathrm{T}))^{3 / 2}$, and the average ion charge number $\mathrm{Z}$ is well approximated by the dependence $\mathrm{Z}$ $(\mathrm{T})^{1 / 2}$. Calculations (see, e.g., [10]) show that, as $T_{e}$ increases from 10 to $100 \mathrm{eV}, \mathrm{Z}$ increases from 6 to 20 ; in this case, $\mathrm{N}_{\mathrm{i}}$ at fixed $\theta$ changes by no more that $10 \%$.

The third reason for the formation of a shadow in photographs is the refraction of the probing beam in plasma regions where the electron density gradient is so large that the refracted rays fall outside the aperture angle of the lens $\alpha=\mathrm{d} / \mathrm{a}$, where $\mathrm{d}$ is the lens diameter and a is the distance from the lens to the object. The deflection angle and the electron-density gradient are related by the formula [11]

$$
\nabla \mathrm{N}_{\mathrm{e}}=-\varepsilon /\left(4.46 \cdot 10^{-14} \lambda^{2} 1\right)
$$

where $\varepsilon$ is the refraction angle, $\lambda$ is the probing-radiation wavelength, and 1 is the plasma length along the probing beam. This formula is valid if the probing-wave frequency is much higher than the frequency of electron - ion (or electron - neutral) collisions $\omega>>\omega_{\text {ei }}$ and much higher than the electron cyclotron frequency, $\omega>>\omega_{\mathrm{e}}=(\mathrm{e} \cdot \mathrm{H}) /(\mathrm{m} \cdot \mathrm{c})$ (here, $\mathrm{H}$ is the magnetic field, and $\mathrm{m}$ is the electron mass), which is always satisfied under experimental conditions.

For the given geometry of the diagnostic windows, the plasma density gradient $\nabla \mathrm{N}_{\mathrm{e}}$ is no less than $3 \cdot 10^{20} \mathrm{~cm}^{-4}$. From the characteristic plasma size observed, we can estimate the electron density as $\mathrm{N}_{\mathrm{e}}=10^{20} \mathrm{~cm}^{-3}$. Consequently, for the tungsten plasma (Fig.4), the ion density can be estimated as $2 \cdot 10^{19} \mathrm{~cm}^{-3}$ and, for other loads, as $5 \cdot 10^{19} \mathrm{~cm}^{-3}$.

Hence, the formation of a shadow in photographs via inverse bremsstrahlung absorption seems to be most probable, at least, for a metal plasma. The appearance of a shadow due to absorption was previously observed in experiments on the heating of foils by focused electron beams [12], as well as in experiments on the implosion of highly emitting gas puffs [13]. This reason is dominant under many experimental conditions.

The refraction angles detected by the schlieren method (Fig.2b) lie in the range from 1 to $6 \mathrm{mrad,}$ which determines the range of detectable density gradients: $10^{19} \mathrm{~cm}^{-4}<\nabla \mathrm{N}_{\mathrm{e}}<5 \cdot 10^{19} \mathrm{~cm}^{-4}$. Taking into account the characteristic plasma size, the electron density is estimated at $10^{19} \mathrm{~cm}^{-3}$.

4. We thank P.I. Blinov for his help in adjustment of the components of the diagnostic complex.

This study was supported by the Russian Foundation for Basic Research (project no. 01-02-17359).

\section{REFERENCES}

1. A.S.Chernenko et al., in Proc. of the $11^{\text {th }}$ Int. Conf. on High-Power Particle Beams, Prague, 1996, p. 154

2. D.V.Vlasov, Zh. Eksp. Teor. Fiz., v.64, p.1986 (1973). [Sov. Phys. JETP, 37, 1001 (1973)].

3. V.A.Gorbunov et al., Izv. Akad. Nauk SSSR, Ser. Fiz., v.48, 1580 (1984).

4. R.R.Buzyalis et al., Kvantovaya Elektron. (Moscow), v.12, 2024 (1985).

5. I.M.Bel'dyuginet al., Kvantovaya Elektron. (Moscow), v.12, 2394 (1985).

6. V.Kh.Bagdasarov et al., Kvantovaya Elektron. (Moscow), v.14, 1364 (1987).

7. V N.Belousov and Yu. K. Nizienko, Preprint No. 4707/7 (Inst. Of At. Energy, Moscow, 1988).

8. R.Alliaga-Rossel et al., in Proc. of the $4^{\text {th }}$ Int. Conf. on Dense Z-pinches, Vancouver, 1997.

9. A.N.Zaydel and G.V.Ostrovskaya, Laser Methods for Plasma Research (Nauka, Leningrad, 1977).

10. D.Mosher, Phys. Rev. v.10, 2330 (1974).

11. E. P.Kruglyakov, Plasma Diagnostics (Atomizdat, Moscow, 1973), issue. 3, p. 97.

12. Yu.M.Gorbulin et al., Preprint No. $4042 / 7$ (Institute of Atomic Energy, Moscow, 1984).

13. S.L.Bogolyubsky et al., in Proc. of the $7^{\text {th }}$ Int. Conf.on High-Power Beams, Karlsruhe, 1988, p.1255.

\section{FIGURE CAPTIONS}

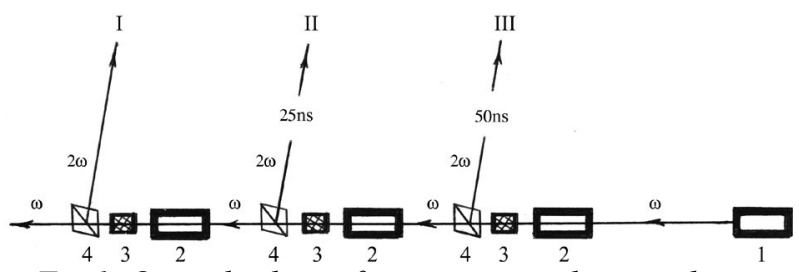

Fig. 1. Optical scheme for generating three probing beams (I, II, and III) with 25 ns delays between the pulses:

1) DO, 2) amplifier, 3) nonlinear crystal for the frequency doubling, and 4) Glan prism for separating the beams at the fundamental and second harmonics. The latter pass through the optical delay lines and are directed into the $S$-300 generator. 


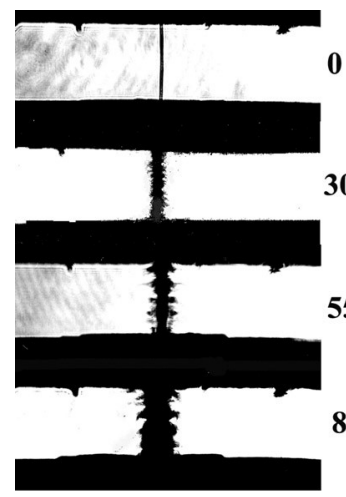

$\mathbf{a}$

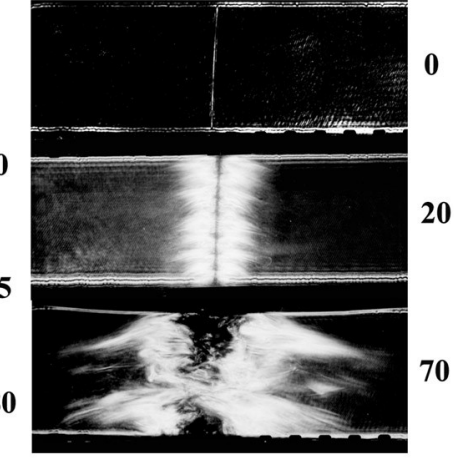

b
Fig.2. (a) shadow photograph of the implosion of an aluminum wire $110 \mu \mathrm{m}$ in diameter and $1 \mathrm{~cm}$ in length at a current through the load of $2 M A$. (b) schlieren photographs of the implosion of a $C_{2} D_{4}$ fiber $30 \mu \mathrm{m}$ in diameter and $1.5 \mathrm{~cm}$ in length at a current of $1.7 \mathrm{MA}$. Schlieren masks allow the detection of the deflection angles of the probing beams in the range from 1 to 6 mrad in all directions. Here and in subsequent figures, numerals on the right from photographs show the delay time (in ns) between the exposure and the current start.

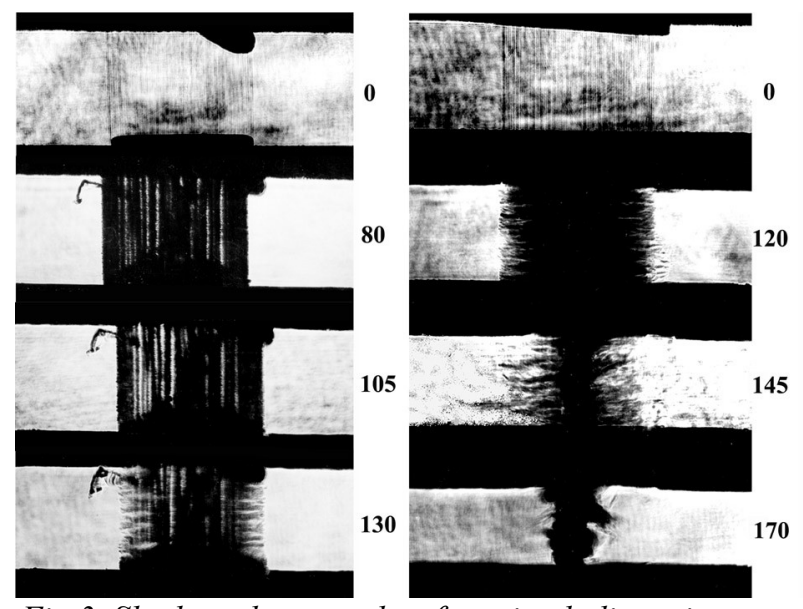

Fig.3. Shadow photographs of two imploding wire arrays composed of eighty tungsten wires $6 \mu \mathrm{m}$ in diameter. The array diameter is $1 \mathrm{~cm}$, its length is $1 \mathrm{~cm}$, the current through the load is 2.6 MA, and the current rise time is $\sim 100$ ns.

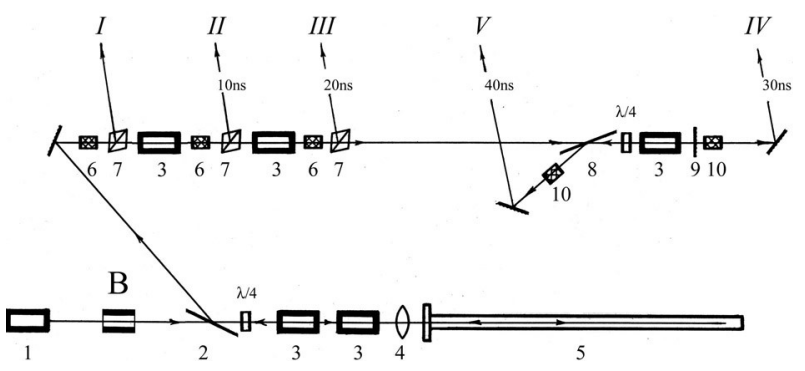

Fig.4. Optical scheme of the transmitting component of the laser diagnostic setup for five-frame plasma probing: 1) $Y A G: N d D O, 2)$, 8) isolators, 3) YAG:Nd amplifiers, 4) lens $(f=0.9 \mathrm{~m}), 5)$ scattering $C C l_{4}$ cell $\left.(l=1.5 \mathrm{~m}), 6\right)$

$\left.\mathrm{SHOs}_{(\mathrm{LiNbO}}\right)$, 7) Glan prisms, 9) mirror with a reflection coefficient of $30 \%$, 10) SHOs (KTP) and B)
Faraday rotator. Five beams pass through the optical delay lines and are directed into the S-300 generator.
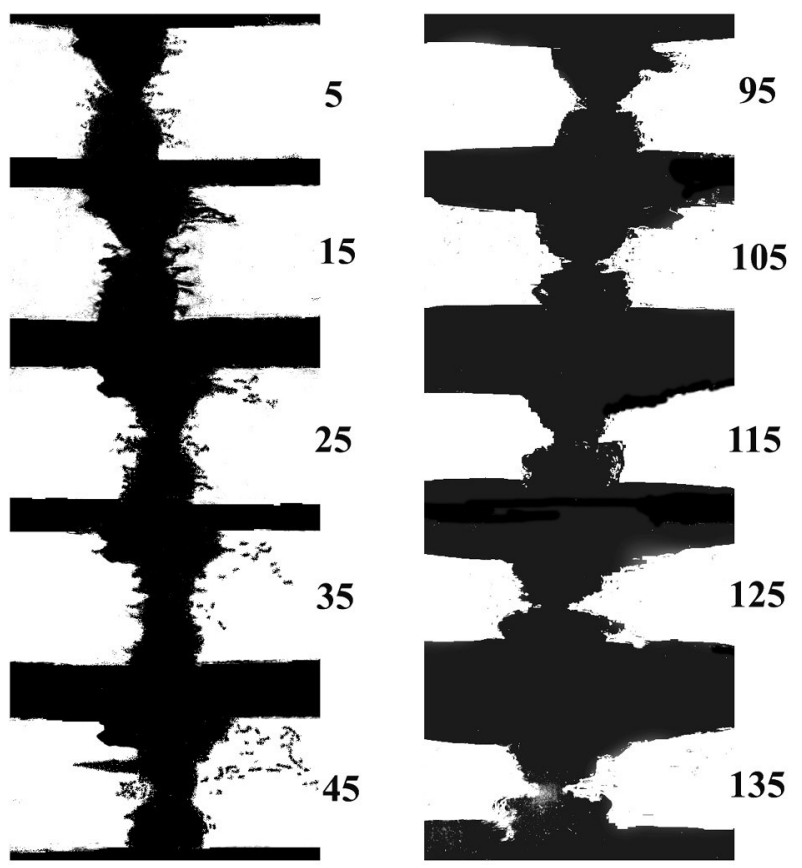

Fig.5. Five-frame shadow photographs of agar-agar Zpinches $5 \mathrm{~mm}$ in diameter and $1 \mathrm{~cm}$ in length. The current through the load is 1.9MA (left frames) and 1.65MA (right frames).
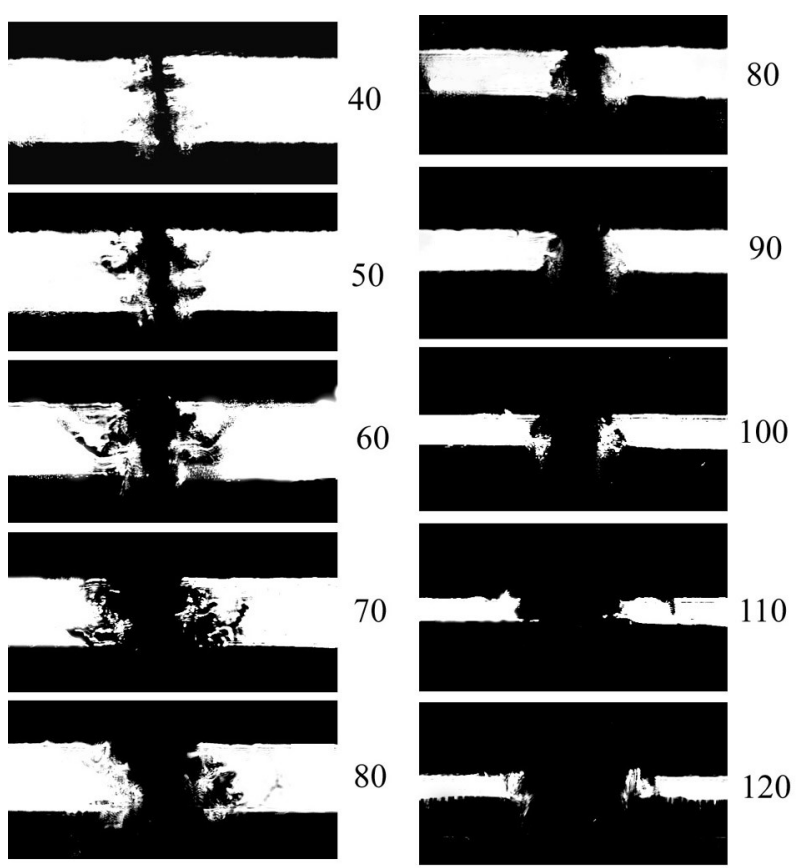

Fig.6. Five-frame shadow photographs of copper wire $120 \mu \mathrm{m}$ in diameter and $0.8 \mathrm{~cm}$ in length at a current through the load of 1.75MA (left frames) and 2.7MA (right frames). 